\title{
Age-depth correlation, grain growth and dislocation-density evolution, for three ice cores
}

\author{
L.W. MORLAND \\ School of Mathematics, University of East Anglia, Norwich NR4 7TJ, UK \\ E-mail: I.morland@uea.ac.uk
}

\begin{abstract}
Two previous theoretical analyses of data from the GRIP, Vostok and Byrd ice cores, presenting age-depth correlations, grain growth and dislocation-density evolution, are re-examined. It is found that the age-depth correlations are inconsistent with the idealized flow with unchanging history adopted, but that good correlations can be obtained by relaxing those restrictions. A modified graingrowth relation is proposed, consistent with the distinct growth profiles of the Vostok and other two cores, which can be solved simultaneously with the given dislocation-density evolution equation. These are solved for all three cores with the given parameters, and the depth profiles of grain diameter and dislocation density at the present time are determined with the new age-depth correlation and with that shown empirically in the papers. The varying flow history influences the age-depth correlation, and hence the depth profiles, which is important both for the interpretation of core data, and for the determination of constitutive variables at each depth at the present time.
\end{abstract}

\section{INTRODUCTION}

Data from ice cores are a crucial element of polar ice study. A variety of physical properties of the ice at each depth are determined, along with its age, which is necessary for the analysis of the evolution of different properties during its passage from the surface to its current location in the core. Here I focus on crystal grain diameter, temperature, dislocation density and strain rate, which are features of fabric evolution and the consequent changing constitutive behaviour of the ice as it descends through the core. In particular, I re-examine the studies of the Greenland Ice Core Project (GRIP) and Vostok (Antarctica) cores of De La Chapelle and others (1998) based on data presented by Thorsteinsson and others (1997) and Lipenkov and others (1989), respectively, and by Montagnat and Duval (2000) who also study the Byrd (Antarctica) core based on data presented by Gow and Williamson (1976). While these papers discuss at length the physical processes occurring in different zones of the core, and how these describe observed behaviour, the present treatment is concerned primarily with solution and modification of the proposed evolution equations, in order to describe the features both of the Vostok core and of the GRIP and Byrd cores, which are distinct. Further, the assumed constant core history over past time is relaxed, allowing a varying surface accumulation and basal melt, and vertical strain rate varying in time, allowing the bed to deform in isostatic equilibrium, in order to correlate observed age and depth. The analyses of De La Chapelle and others (1998) and Montagnat and Duval (2000) assume a compressive vertical strain rate (implicitly with a lateral strain rate identical in all directions to satisfy incompressibility), which is uniform down the core, and that this strain rate, the depth, the surface and bed conditions (and so the ice thickness) and the temperature have remained steady through the past time.

The schematic behaviour presented in the above papers shows, in the GRIP and Byrd cores, a near-surface zone, to a depth of hundreds of metres, in which there is normal grain growth until an equilibrium grain diameter is reached, then a zone of rotation (continuous) recrystallization with uniform grain diameter in which polygonization counteracts the growth, then a shallow basal zone at higher temperature of migration (discontinuous) recrystallization in which the grain diameter increases and the fabric weakens and isotropy is possibly restored. In the Vostok core, unlike the others, the temperature increases steadily with depth from the surface, as does the grain diameter. My analysis uses only data points taken from the growth curves presented in these papers, not actual observation data. A critical review of these recrystallization processes, and the theoretical modelling, has been presented by Placidi and others (2006), who also refer to migration recrystallization as dynamic recrystallization. The papers and the present analysis treat only the grain growth and dislocation-density evolution equations prior to the onset of migration recrystallization, but the state at this point is what triggers the migration recrystallization, and is therefore a feature in the constitutive modelling of subsequent migration recrystallization. Two preliminary constitutive theories for such weakening and loss of fabric have been proposed by Staroszczyk and Morland (2001) and Morland (2002), but their deficiencies have been noted by Placidi and others (2006).

Let $t$ denote time, $T$ denote temperature, $D$ denote the mean grain diameter and $\zeta$ denote the dislocation density. During the initial normal growth, $D$ increases from an initial (surface) value, $D_{0}$, to an equilibrium value, $D_{\mathrm{e}}$, in the GRIP and Byrd cores, governed by (Stevenson, 1967; Gow, 1969)

$$
\frac{\mathrm{d} D^{2}}{\mathrm{~d} t}=K(T), \quad \frac{\mathrm{d} D}{\mathrm{~d} t}=\frac{K(T)}{2 D},
$$

where $K(T)$ is the grain-boundary migration rate, which is an increasing function of $T$. The growth relation (1) is that used by De La Chapelle and others (1998) and Montagnat and Duval (2000). While this applies during the initial growth in the Vostok core, it is suggested that growth continues without reaching an equilibrium value, due to the increase of $T$, and hence of $K(T)$, but no growth law is presented for the rotational recrystallization stage which leads to an equilibrium value for the GRIP and Byrd cores, but increasing 
Table 1. Core parameters

\begin{tabular}{lccccccc}
\hline Core & $\begin{array}{c}D_{0} \\
\mathrm{~mm}\end{array}$ & $\begin{array}{c}D_{\mathrm{e}} \\
\mathrm{mm}\end{array}$ & $\begin{array}{c}h_{\mathrm{c}} \\
\mathrm{m}\end{array}$ & $\begin{array}{c}s_{0} \\
\mathrm{ka}^{-1}\end{array}$ & $\begin{array}{c}T_{\mathrm{s}} \\
{ }^{\circ} \mathrm{C}\end{array}$ & $\begin{array}{c}K_{\mathrm{s}} \\
\mathrm{m}^{2} \mathrm{ka}^{-1}\end{array}$ & $\begin{array}{c}\zeta_{0} \\
\mathrm{~m}^{-2}\end{array}$ \\
\hline Byrd & 1.575 & 6.3 & 2160 & 0.0788 & -28 & $1.104 \times 10^{-5}$ & $10^{10}$ \\
GRIP & 1 & 4.0 & 3030 & 0.0788 & -32 & $0.378 \times 10^{-5}$ & $10^{10}$ \\
Vostok & 1 & & 3350 & 0.00725 & -56 & & $10^{10}$ \\
\hline
\end{tabular}

Notes: $D_{0}$ is initial grain diameter, $D_{\mathrm{e}}$ is equilibrium diameter, $h_{\mathrm{c}}$ is core length, $s_{0}$ is initial strain rate, $T_{\mathrm{s}}$ is surface temperature, $K_{\mathrm{s}}$ is surface migration rate and $\zeta_{0}$ is initial dislocation density.

$D$ in the Vostok core. Jacka and Li (1994) proposed the more general form

$$
\frac{\mathrm{d} D}{\mathrm{~d} t}=\frac{K}{D}-P D,
$$

with the second term with positive coefficient $P$ reducing the growth rate. Placidi and others (2006) give the solution for constant $K$ and $P$ and illustrate how $D$ asymptotes to an equilibrium value at a rate defined by $P$. Here a more general modification is deduced which leads to the equilibrium value of $D$ at finite $t$ for the GRIP and Byrd conditions, but reproduces the continuous increase for the Vostok conditions.

A reviewer has pointed out that a normal growth relation is only applicable below any melt zone, so that $D_{0}$ must be interpreted as the mean diameter at the interface with the fully frozen zone, and that location treated as the surface. Furthermore, while $D_{0}$ is a necessary initial value for the differential equation (Equation (1) or (2)), surface observations do not yield a clear-cut value, so the grain diameter growth given by integrating the differential equation (Equation (1) or (2)) from an assumed $D_{0}$ is suspect even over short times and modest depths. However, the proposed modifications to describe the longer-time, deeper-diameter profiles automatically correlate with better-measured mean diameters, and are less sensitive to the choice of $D_{0}$. Furthermore, Gao and Jacka (1987) and Jacka and Li (1994) emphasize that the equilibrium diameter is independent of $D_{0}$. The present comparison calculations adopt values of $D_{0}$ inferred from the figures of De La Chapelle and others (1998) and Montagnat and Duval (2000), presumably the initial values for their solutions.

The evolution of dislocation density from an initial value, $\zeta_{0}$, at the surface is governed by (De La Chapelle and others, 1998; Montagnat and Duval, 2000)

$$
\frac{\mathrm{d} \zeta}{\mathrm{d} t}=\frac{s}{b_{\mathrm{v}} D}-\alpha \frac{K(T) \zeta}{D^{2}}
$$

where $s$ is the compressive vertical strain rate, $b_{v}$ is the Burgers vector and $\alpha$ is a parameter which is not less than unity. The previous illustrations were mainly with $\alpha=1$, but Montagnat and Duval (2000) illustrate dislocation-energy evolution for the GRIP core with constant $K$ for $\alpha=1,2$ and 3 , to conclude that $\alpha=3$ leads to a too low dislocation density for the transition to rotation recrystallization, and $\alpha$ between 2 and 3 would lead to an equilibrium dislocation density close to the depth where grain growth reaches its equilibrium value.

Solution of evolution equations from past ice deposit times at the surface to the present (core observation) time

$$
\begin{aligned}
& z=\underline{h}(t) \ldots \_q(t) \ldots \ldots \\
& z=h_{0}
\end{aligned}
$$

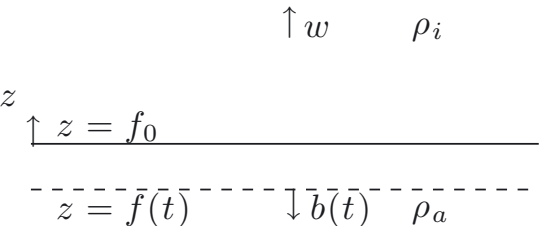

Fig. 1. Bed, $f$, and surface, $h$, at present time, $t=0$, and at past time, $t$.

requires knowledge of the history of the ice-flow variables, not just present values, when the core is not assumed to have been in its present state since the ice was deposited. Retaining the assumption of vertical strain rate uniform in depth, a more general history is allowed, as described above, to obtain good age-depth correlations for the three cores, by a least-squares calculation with selected data points, showing that the non-changing-history assumption with the given parameters is not consistent with the presented data. The grain-growth equation is then modified to be consistent with the distinct core observations, and comparisons made with the original solutions and observations. Finally, the same dislocation-energy evolution equation is solved with the new and original grain growth to illustrate the different behaviours.

Table 1 lists the parameters given by De La Chapelle and others (1998) and Montagnat and Duval (2000) and inferred from their figures, which are adopted here, together with $b_{\mathrm{v}}=4.5 \times 10^{-10} \mathrm{~m}$ (De La Chapelle and others, 1998).

The inferred initial grain diameters, $D_{0}$, for all three cores are chosen to be one-quarter of the given $D_{\mathrm{e}}$ for the Byrd and GRIP cores, which appear to be good approximations from the figures, and allow a simplified common analysis; it is not a necessary restriction; $h_{\mathrm{c}}$ is the core length. The temperature in the Byrd and GRIP cores remains at the surface temperature, $T_{\mathrm{S}}$, for a large fraction of the core length, and the later illustrations adopt a fraction 2/3, followed by a smooth quadratic increase to a basal temperature of $-10^{\circ} \mathrm{C}$. For the Vostok core, a quadratic increase from the surface temperature to the same basal temperature is adopted, with decay length adjusted to match a feature of the profile shown by De La Chapelle and others (1998). The grain-boundary migration rate dependence on temperature is discussed below, in particular how it matches the surface values, $K_{\mathrm{s}}$, for the Byrd and GRIP cores and applies to the temperature range of the Vostok core. The time unit ka is chosen as a practical scale.

\section{AGE-DEPTH CORRELATION}

Figure 1 shows schematic bed $(z=f(t))$ and surface $(z=$ $h(t))$ positions with respect to a vertically upward coordinate, $z$, with origin at the present bed position, $f_{0}=f(0)=0$, where $t=0$ is the present time with the surface at $z=$ $h(0)=h_{0}$. Motion in the core is assumed to be purely vertical, with upward velocity $w$. The surface is subject to an accumulation (inward ice flux per unit area) $q(t)$, assumed positive, with present value $q_{0}$, and the bed is subject to 
melting (outward ice flux per unit area) $b(t)$, of either sign, with present value $b_{0}$. The ice density is $\rho_{\mathrm{i}}=917 \mathrm{~kg} \mathrm{~m}^{-3}$, and the asthenosphere below is assumed to be a viscous fluid with density $\rho_{\mathrm{a}}=3300 \mathrm{~kg} \mathrm{~m}^{-3}$ (Greve, 2001). Isostatic equilibrium is assumed over all past time; that is

$$
\rho_{\mathrm{i}}\left(h-f-h_{0}\right)=\rho_{\mathrm{a}}(-f),
$$

which implies

$$
f=\frac{\rho_{\mathrm{i}}}{\rho_{\mathrm{a}}}\left(h_{0}-\Delta\right), \quad h=\frac{\rho_{\mathrm{i}}}{\rho_{\mathrm{a}}} h_{0}+\frac{\rho_{\mathrm{a}}-\rho_{\mathrm{i}}}{\rho_{\mathrm{a}}} \Delta,
$$

where the ice thickness is

$$
\Delta(t)=h(t)-f(t), \quad \Delta_{0}=\Delta(0)=h_{0} .
$$

The surface and bed accumulation and melting conditions are

$$
z=h(t): \quad \frac{\mathrm{d} h}{\mathrm{~d} t}=w_{\mathrm{s}}+q, \quad z=f(t): \quad \frac{\mathrm{d} f}{\mathrm{~d} t}=w_{\mathrm{b}}+b,
$$

where subscripts 's' and ' $b$ ' denote values on the surface and bed, respectively. The assumption of a vertical compressive strain rate, $s(t)$, uniform in $z$ implies

$$
\begin{gathered}
s(t)=-\frac{\mathrm{d} w}{\mathrm{~d} z}, \quad w(z, t)=w_{\mathrm{b}}(t)-s(t)[z-f(t)], \\
w_{\mathrm{s}}-w_{\mathrm{b}}=-s(t) \Delta(t),
\end{gathered}
$$

and hence from the boundary conditions, Equations (7),

$$
\frac{\mathrm{d} \Delta}{\mathrm{d} t}+s(t) \Delta=q-b=\bar{q}(t) .
$$

Define the integrating factor for the differential equation (9):

$$
J(t)=\exp \left[-\int_{t}^{0} s(\tau) \mathrm{d} \tau\right], \quad 1 \geq J>0 \quad(t \leq 0),
$$

then

$$
J^{\prime}(t)=s(t) J(t), \quad \int_{t}^{0} s(\tau) J(\tau) \mathrm{d} \tau=1-J(t),
$$

and the solution of Equation (9) is

$$
J(t) \Delta(t)=\Delta_{0}-Q(t), \quad Q(t)=\int_{t}^{0} J(\tau) \bar{q}(\tau) \mathrm{d} \tau,
$$

$Q(0)=0, \quad Q^{\prime}(t)=-J(t) \bar{q}(t)>0, \quad Q>0 \quad(t<0)$,

and $f(t)$ and $h(t)$ are then determined by the relations in Equation (5).

Let $\mathrm{P}$ be the ice particle deposited at the surface, $z=h\left(t_{\mathrm{p}}\right)$, at a time $t_{p} \leq 0$, which reaches position $z\left(t_{p}\right)$ at the present time, $t=0$. Then $\mathrm{P}$ has a path, $z=z_{\mathrm{p}}(t)$, given by

$$
\begin{array}{r}
\frac{\mathrm{d} z_{\mathrm{p}}}{\mathrm{d} t}=w\left[z_{\mathrm{p}}(t), t\right]=w_{\mathrm{b}}(t)-s(t)\left[z_{\mathrm{p}}(t)-f(t)\right], \\
z_{\mathrm{p}}\left(t_{\mathrm{p}}\right)=h\left(t_{\mathrm{p}}\right), \quad\left(t_{\mathrm{p}} \leq t \leq 0\right),
\end{array}
$$

which has the same integrating factor, $J(t)$, as Equation (9). Integrating from $t=t_{\mathrm{p}}$ to $t=0$ gives

$$
z\left(t_{\mathrm{p}}\right)=J\left(t_{\mathrm{p}}\right) h\left(t_{\mathrm{p}}\right)+\int_{t_{\mathrm{p}}}^{0} J(\tau)\left[w_{b}(\tau)+s(\tau) f(\tau)\right] \mathrm{d} \tau .
$$

Now differentiating Equation (15) and eliminating $h^{\prime}\left(t_{\mathrm{p}}\right)$ by Equation (7), $J^{\prime}\left(t_{p}\right)$ by Equation (11) and the resulting $w_{\mathrm{s}}\left(t_{\mathrm{p}}\right)-w_{\mathrm{b}}\left(t_{\mathrm{p}}\right)$ by Equation $\left(8_{3}\right)$ gives

$$
\frac{\mathrm{d} z}{\mathrm{~d} t_{\mathrm{p}}}=J\left(t_{\mathrm{p}}\right) q\left(t_{\mathrm{p}}\right)>0
$$

That is, $z\left(t_{\mathrm{p}}\right)$ is strictly increasing, with a unique inverse, $t_{p}(z)$, as physically required. Integrating the differential equation (16) from $t=t_{\mathrm{p}}$ to $t=0$ now leads to a much simpler expression than Equation (15) for $z\left(t_{p}\right)$ :

$$
z\left(t_{\mathrm{p}}\right)=h_{0}-\int_{t_{\mathrm{p}}}^{0} J(\tau) q(\tau) \mathrm{d} \tau,
$$

depending only on the surface accumulation, and not on the basal melting; again as physically required. In terms of the depth, $\bar{z}$, and age, $\bar{t}$, of $\mathrm{P}$ in the present core,

$$
\bar{z}=h_{0}-z=\int_{0}^{\bar{t}} J(-\bar{\tau}) q(-\bar{\tau}) \mathrm{d} \bar{\tau}, \quad \bar{t}=-t_{\mathrm{p}} .
$$

With the core assumptions of De La Chapelle and others (1998) and Montagnat and Duval (2000), $s \equiv s_{0}, f \equiv 0$, $h \equiv h_{0}$, and hence by Equation (9), $\bar{q} \equiv \bar{q}_{0} \equiv s_{0} h_{0}$, and implicitly $q \equiv q_{0}$ and $b \equiv b_{0}$. Set $b_{0}=r q_{0}, r<1$, then Equations (10) and (18) give the simple age-depth correlation

$$
J(t)=\exp \left(s_{0} t\right), \quad \frac{\bar{z}}{h_{0}}=\frac{1}{1-r}\left[1-\exp \left(-s_{0} \bar{t}\right)\right] .
$$

Later illustrations adopt $b=0$, so $r=0$, but non-zero $r$ simply applies a common scale factor to $\bar{z}$ at each $\bar{t}$.

Anticipating that this correlation does not match the empirical data presented by De La Chapelle and others (1998) and Montagnat and Duval (2000) for the three cores, the general relation, Equation (18), is now matched to selected data points for the three cores by least-squares correlation using simple positive functions, $s(t)$ and $q(t)$, with five unknown parameters. The improved correlations analogous to the result, Equation (19), but allowing a different constant $s_{0}$, are also determined. Let

$$
\begin{gathered}
s(t)=s_{0} \tilde{s}(y), \quad q(t)=q_{0} \tilde{q}(y), \quad y=s_{0} t<0, \\
\tilde{s}(y)=\exp \left(s_{1} y+s_{2} y^{2}\right), \quad \tilde{q}(y)=\exp \left(q_{1} y+q_{2} y^{2}\right),
\end{gathered}
$$

which ensure positive $s$ and $q$. Here $s_{0}$ is given, but $q_{0}$ is a free parameter along with $\left[s_{i}, q_{i}(i=1,2)\right]$, giving five free parameters. Now Equations (10) and (18) are expressed by

$$
\begin{array}{r}
J(t)=\tilde{\jmath}(y)=\exp \left[-\int_{y}^{0} \tilde{s}(\tilde{y}) \mathrm{d} \tilde{y}\right], \\
\frac{\bar{z}}{h_{0}}=R \int_{-\bar{y}}^{0} \tilde{\jmath}(\tilde{y}) \tilde{q}(\tilde{y}) \mathrm{d} \tilde{y}, \quad R=\frac{q_{0}}{s_{0} h_{0}}, \quad \bar{y}=s_{0} \bar{t},
\end{array}
$$

so parameter $q_{0}$ enters only in the multiplying factor, $R$, which is treated as one of the five parameters in the leastsquares correlation to satisfy Equation (22).

The parameter $h_{0}$, the current depth and thickness, enters the isostasy relations, Equations (4-6), and the consequent linear differential equation (9) for the thickness over past time. It is specifically the current value of the thickness (Equation $\left.\left(6_{2}\right)\right)$, and any change in $h_{0}$ simply makes the same additive change to $\Delta(t)$. However, the $\bar{z} / h_{0}$ relation to $\bar{t}$ in Equation (22) involves $h_{0}$ only in the denominator of the factor $R$, and so does not appear in the actual $\bar{z}-\bar{t}$ relation. It is therefore just a convenient normalizing factor for the purpose of contrasting the different correlations, and the simple choice $h_{0}=h_{\mathrm{c}}$, the core length, is made, so each core has a common span $0 \leq \bar{z} / h_{0} \leq 1$. Note, though, that the kinematic conditions (Equation (7)) involve actual surface and bed positions, so this choice of $h_{0}$ is applying the basal melt, $b$, at the core base, and not at the actual bed. 


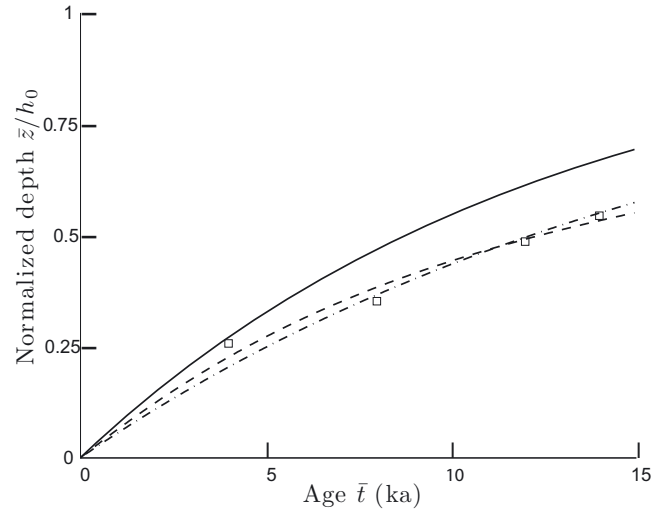

Fig. 2. Byrd core: age-normalized depth correlations. Squares are given points; solid curve is given $s_{0}$ solution; dot-dashed curve is optimum $s_{0}$ solution; dashed curve is correlated solution.

Hence the term $\bar{q}$, which enters the thickness equation (9) and correlation relation (22), now refers to the change over the core length and not the depth. The choice, $b=0$, made for the illustrations could be just as good an approximation at the core base as at the bed.

The selected depth-age points for the Byrd and Vostok cores are taken from the figures of Montagnat and Duval (2000) and De La Chapelle and others (1998), respectively, and a selection from both for the GRIP core, marked as squares in Figures 2-4, covering time ranges 15, 50 and $300 \mathrm{ka}$, respectively. As constructed, the general correlation closely matches the given points for each core. The given $s_{0}$ correlation, Equation (19), with $r=0$ is reasonable for the Vostok core, but not the Byrd and GRIP cores, and applying a common $\bar{z}$ scaling at each $\bar{t}$ by changing the melting proportionality, $r$, would not yield a good correlation. The figures also show the correlation, Equation (19), with an optimum constant strain rate, $s_{\mathrm{d}} s_{0}$, instead of $s_{0}$, for each core, where

$$
\begin{array}{r}
\text { Byrd : } s_{\mathrm{d}}=0.722, \quad \text { GRIP }: s_{\mathrm{d}}=0.755, \\
\text { Vostok }: s_{\mathrm{d}}=0.856 .
\end{array}
$$

These optimum constant strain rates are all appreciably smaller than the given $s_{0}$, and now both Byrd and Vostok correlations are reasonable, but not the GRIP correlation. Figures 5-7 show the variation of $h, f, q$ and $s$ with past time, $\bar{t}$, corresponding to the general correlations for the three cores. The values of $q_{0}$ determined by parameter $R$, with the

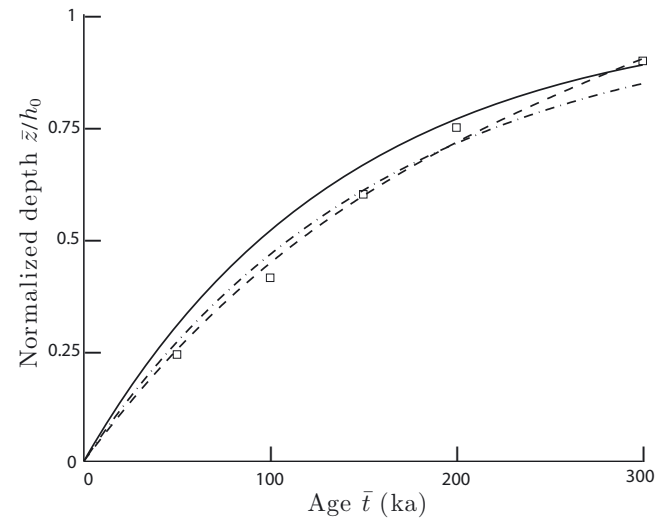

Fig. 4. Same as Figure 2, but for Vostok core.

given $s_{0}$, for the three cores are:

$$
\begin{aligned}
\text { Byrd }: q_{0}=0.140 \mathrm{ma}^{-1}, \text { GRIP }: q_{0} & =0.215 \mathrm{ma}^{-1}, \\
\text { Vostok }: q_{0} & =0.019 \mathrm{~m} \mathrm{a}^{-1},
\end{aligned}
$$

which would decrease in proportion for an alternative, lower, $s_{0}$ such as those given by the optimum values (Equation (23)). No correlations could be found for the case $q \equiv q_{0}$, a free parameter, with varying $s(t)$. In the case $s \equiv s_{0}$ with varying $q(t)$, an enormous thickness in the past was predicted for the GRIP core, increasing as $r$ was increased, and in the case $s \equiv s_{0}, f \equiv 0$ (no bed deformation), the surface elevation, $h$, in the past was even higher. The best correlations obtained for the three cores were, therefore, the case of varying $s(t), q(t)$ and bed deformation, $f(t)$, satisfying isostasy.

Note, however, that the predicted variations of $q$ and $s$ for the GRIP core with the general correlation are rather dramatic. Given more information about past variations of various parameters, different correlation structures could be determined. The present one is used to illustrate how past history enters the evolution equations, and its possible influence on predictions. These predictions hinge on the underpinning assumptions that the vertical strain rate is, and was during the period of application, uniform with depth, and that the ice velocity in the core has been essentially vertical. While these may be unsatisfactory, solution of the evolution equations for grain growth and dislocation density from the initial deposit time, and their display as profiles

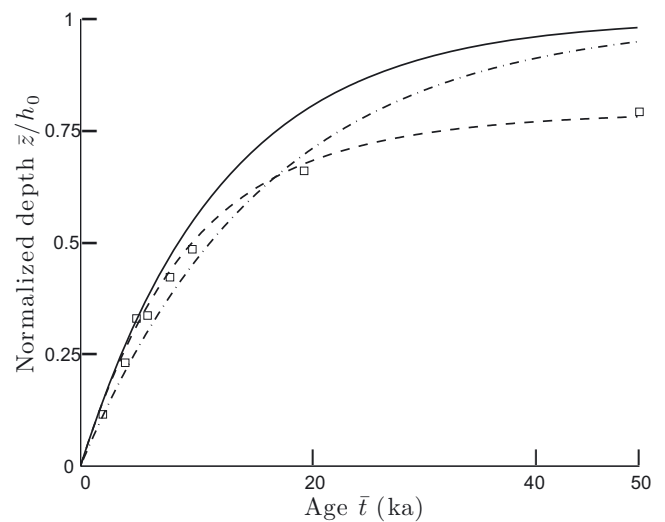

Fig. 3. Same as Figure 2, but for GRIP core.

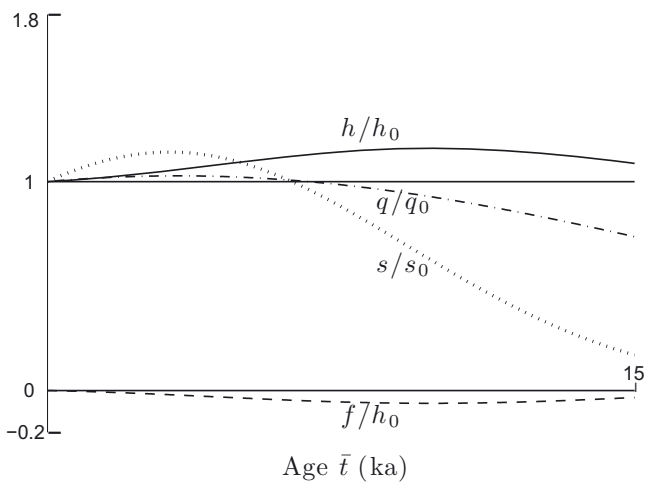

Fig. 5. Byrd core: bed, $f$, surface, $h$, strain rate, $s$, and surface accumulation, $q$, as normalized variables at age $\bar{t}$. 


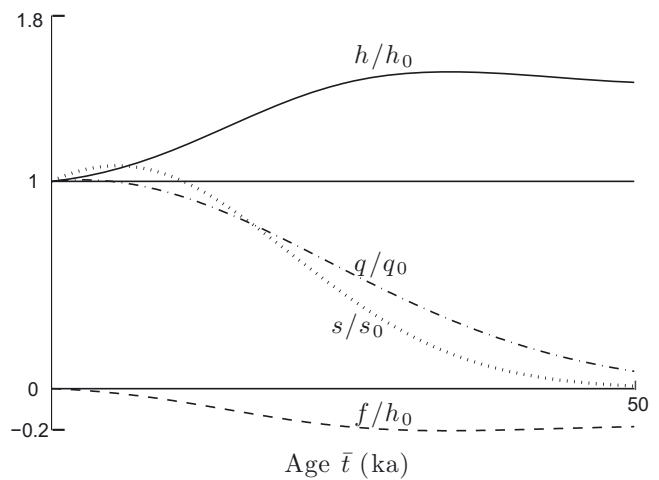

Fig. 6. Same as Figure 5, but for GRIP core.

with depth at the present time, require a consistent agedepth correlation that is not given by Equation (19). In the later solutions, general correlation refers to the correlation given by Equations (20-22), and previous correlation to Equation (19) with the given $s_{0}$.

\section{TEMPERATURE AND GRAIN-BOUNDARY MIGRATION RATE}

In the Byrd and GRIP cores the temperature remains constant to a large depth, but in the Vostok core the temperature increases steadily with depth. Introducing a normalized dimensionless temperature, $\bar{T}$, by

$$
T=T_{0}+\left[20^{\circ} \mathrm{C}\right] \bar{T}, \quad T_{0}=0^{\circ} \mathrm{C},
$$

which is of order unity over typical ice-sheet temperature ranges, melting down to $60^{\circ} \mathrm{C}$ below melting. The surface temperatures for the three cores (Table 1 ) are then given by

$$
\begin{array}{r}
\text { Byrd : } \bar{T}_{\mathrm{s}}=-1.4, \quad \text { GRIP }: \bar{T}_{\mathrm{s}}=-1.6, \\
\text { Vostok }: \bar{T}_{\mathrm{s}}=-2.8 .
\end{array}
$$

The temperature profiles in $\bar{z}$ for the present time in the Byrd and GRIP cores are modelled as constant down to a fraction $(1-p)$ of the core, then by a quadratic with continuous derivative reaching a temperature $T_{\mathrm{b}}=-10^{\circ} \mathrm{C}, \bar{T}_{\mathrm{b}}=-0.5$, at the base, with $p=1 / 3$ adopted for illustrations. For the Vostok core, the entire temperature profile in $\bar{Z}$ is modelled by a quadratic increasing from $\bar{T}=-2.8$ at the surface to $\bar{T}=-0.5$ at the base, with the temperature gradient ratio between bed and surface, $\omega$, set to 4 to approximate the figure shown in De La Chapelle and others (1998). That is, for Byrd and GRIP,

$$
\begin{array}{r}
\bar{T}(\bar{z})=\bar{T}_{\mathrm{s}} \quad\left(0 \leq \bar{z} / h_{0} \leq 1-p\right), \\
\bar{T}(\bar{z})=\bar{T}_{\mathrm{s}}+\left(\bar{T}_{\mathrm{b}}-\bar{T}_{\mathrm{s}}\right)\left(\bar{z} / h_{0}-1+p\right)^{2} / p^{2} \\
\left(1-p \leq \bar{z} / h_{0} \leq 1\right),
\end{array}
$$

and for Vostok, with $\omega=0$,

$$
\bar{T}(\bar{z})=\bar{T}_{\mathrm{s}}+\frac{2\left(\bar{T}_{\mathrm{b}}-\bar{T}_{\mathrm{s}}\right)}{1+\omega}\left[\frac{\bar{z}}{h_{0}}+\frac{\omega-1}{2}\left(\frac{\bar{z}}{h_{0}}\right)^{2}\right] .
$$

These are shown as profiles of the normalized depth, $\bar{z} / h_{0}$, against the physical temperature, $T$, in Figure 8 , and the same functions of depth with respect to varying $h(t)$ in the general age-depth correlation are adopted in the evolution equations through past time.

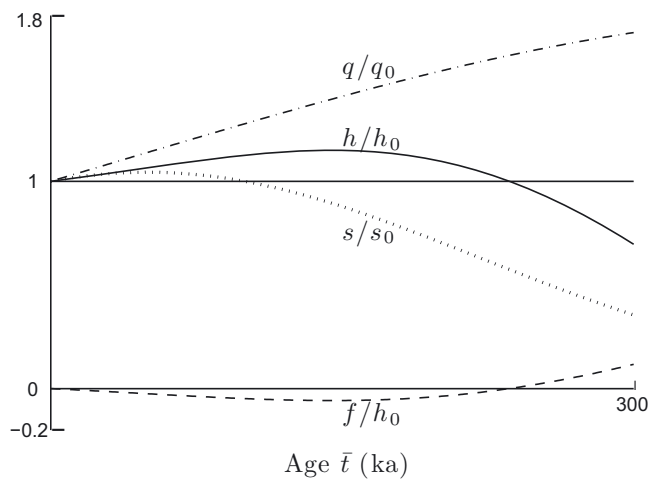

Fig. 7. Same as Figure 5, but for Vostok core.

The grain-boundary migration rate, $K(T)$, is an increasing function of temperature (De La Chapelle and others, 1998), and hence of $\bar{T}$, though no details of its dependence are presented. Surface temperatures for the three cores are shown in Table 1, with dimensionless values given in Equation (26), but $K_{S}$ was given only for Byrd and GRIP, and decreases significantly between $\overline{T_{\mathrm{s}}}=-1.4$ and $\overline{T_{\mathrm{s}}}=$ -1.6 . Lacking further information, it is supposed that $K$ does not change dramatically from the Byrd and GRIP surfacetemperature values at lower and higher temperatures. Let $K \rightarrow K_{\mathrm{c}}$ at very low temperature, less than $\bar{T}_{\mathrm{s}}=-2.8$, then these conditions are satisfied by a function

$$
K(\bar{T})=K_{\mathrm{C}}+0.5 \kappa\{1+\tanh [\gamma(\bar{T}+1.5)]\},
$$

with

$$
\begin{array}{r}
K_{\mathrm{c}}=0.1 \times 10^{-5} \mathrm{~m}^{2} \mathrm{ka}^{-1}, \\
\kappa=1.28 \times 10^{-5} \mathrm{~m}^{2} \mathrm{ka}^{-1} \quad \text { and } \gamma=6.365,
\end{array}
$$

for which, to a very close approximation,

$$
K(-2.8)=K_{\mathrm{c}}, \quad K(-0.5)=K(0)=1.38 \times 10^{-5} \mathrm{~m}^{2} \mathrm{ka}^{-1} .
$$

This form, chosen only to be consistent with the values given, and not physically derived, is adopted for the new correlation illustrations. For solutions with the previous correlation, $K$ is kept constant at the respective surface values given in Table 1 and Equation $\left(31_{1}\right)$, since no variation was indicated.

\section{GRAIN GROWTH AND DISLOCATION-DENSITY EVOLUTION}

In the Byrd and GRIP cores, while the temperature remains constant to a large depth, the grain diameter first grows to an equilibrium value, with growth rate depending on temperature, described by Equation (1), then remains constant for most of the core depth. In the Vostok core, both the temperature and grain diameter increase continuously, with no equilibrium diameter reached. The dislocation-density evolution, described by differential equation (3), depends on the temperature, strain rate and grain diameter. Introducing a normalized dimensionless grain diameter, $\bar{D}$, and dimensionless dislocation density, $\bar{\zeta}$, by

$$
D=D_{0} \bar{D}, \quad \zeta=\zeta_{0} \bar{\zeta},
$$

where the initial values, $D_{0}$ and $\zeta_{0}$, are given in Table 1 , then the equilibrium grain diameters for the Byrd and GRIP cores 


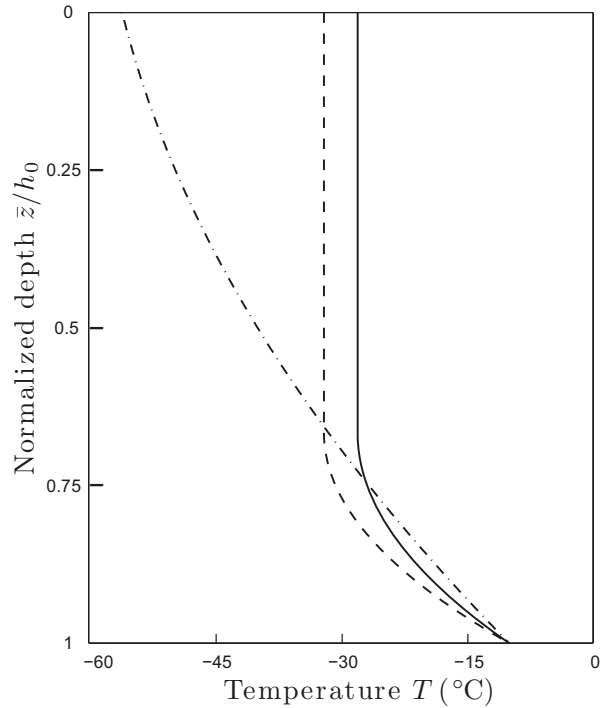

Fig. 8. Normalized-depth-physical-temperature $(T)$ profiles for Byrd (solid curve), GRIP (dashed curve) and Vostok (dot-dashed curve) cores.

are both given by $\bar{D}_{\mathrm{e}}=4$, but this is not a restriction on the analysis. Let $\tilde{t}$ be the time spent in the core at time $t$ by a particle $\mathrm{P}$ deposited at time $t_{\mathrm{p}}$, then

$$
\tilde{t}=t-t_{\mathrm{p}}, \quad \bar{D}=\bar{\zeta}=1 \text { at } \tilde{t}=0 .
$$

The grain-growth and dislocation-energy equations (1) and (3) for particle $\mathrm{P}$, for $D$ and $\zeta$, respectively, become

$$
\begin{gathered}
\frac{\mathrm{d} \bar{D}}{\mathrm{~d} \tilde{t}}=\frac{K[\bar{T}(\bar{z})]}{2 D_{0}^{2} \bar{D}}, \\
\frac{\mathrm{d} \bar{\zeta}}{\mathrm{d} \tilde{t}}=\frac{s_{0} \tilde{s}\left[s_{0}\left(\tilde{t}+t_{\mathrm{p}}\right)\right]}{b_{\mathrm{v}} \zeta_{0} d_{0} \bar{D}}-\frac{\alpha K[\bar{T}(\bar{z})] \bar{\zeta}}{D_{0}^{2} \bar{D}^{2}},
\end{gathered}
$$

in the dimensionless variables, where the depth, $\bar{z}$, is that of particle $\mathrm{P}$ at age $\tilde{t}$, given by Equation (18) for general correlation, and by Equation (19) for the previous correlation, with $\bar{t}=\tilde{t}$. The initial values, $D_{0}$ and $\zeta_{0}$, now appear in the dimensionless evolution equations instead of the initial conditions, but note that differential equations (34) and (35) are equivalent to the physical rate laws, Equations (1) and (3), which do not depend on the initial values.

First consider grain growth for the Byrd and GRIP cores where $\bar{D}_{\mathrm{e}}$ is reached after a finite time, at different constant temperatures, and then remains constant. This is not realized by the differential equation (2), and a different modification to Equation (1) is required. An appropriate modification to the corresponding dimensionless form, Equation (34), with a different structure to Equation (2), is

$$
\frac{\mathrm{d} \bar{D}}{\mathrm{~d} \tilde{t}}=\frac{K[\bar{T}(\bar{z})]}{2 D_{0}^{2} \bar{D}}[1-g(\bar{D})],
$$

such that the constitutive function, $g(\bar{D})$, is close to zero for $\bar{D}<\bar{D}_{\text {e, }}$, increases continuously to near unity over a small range of $\bar{D}$ centred on $\bar{D}_{\mathrm{e}}$, then remains approximately unity. The common temperature-dependent factor is necessary so that the $\bar{D}$ growth can be switched off beyond $\bar{D}_{\mathrm{e}}$,

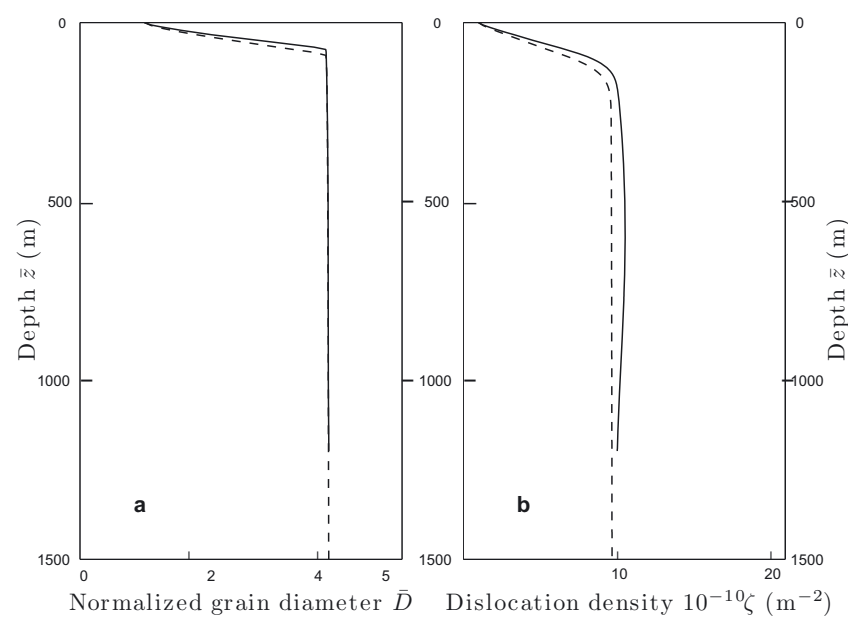

Fig. 9. Byrd core: depth profiles at $t=0$ of (a) normalized grain diameter, $\bar{D}$, and (b) dislocation density, $\zeta$. New correlation solutions (solid curves); solutions for grain growth, Equation (41), with given $s_{0}$ and constant $K$ (dashed curves).

independent of the temperature. The function adopted for illustrations is

$$
g(\bar{D})=0.5\left\{1+\tanh \left[d\left(\bar{D}-\bar{D}_{\mathrm{e}}\right)\right]\right\}, \quad d=100 ;
$$

the large parameter, $d$, determines the rapid change range. Differential equation (36) with function (37) is virtually Equation (1) until $\bar{D}_{\mathrm{e}}$ is reached, then ensures negligible further growth. The term with factor $g(\bar{D})$ provides the necessary slowing of the growth rate, consistent with observation, but this form has no physical basis.

For the Vostok core, the grain growth is not switched off at $\bar{D}_{\mathrm{e}}$, nor at larger $\bar{D}$, and the increasing temperature does not prevent the $[1-g(\bar{D})]$ factor in Equation (36) from approaching zero beyond $\bar{D}_{\mathrm{e}}$. What is different for Vostok is its much lower vertical compressive strain rate, $s$. For constitutive purposes we measure strain rate by the second principal invariant, $I$, of the (deviatoric) strain rate, $\boldsymbol{D}$, given by

$$
I=\frac{1}{2} \operatorname{tr} \boldsymbol{D}^{2}=3 s^{2} / 4
$$

in the assumed core deformation, and introducing a magnitude $I^{\star}$ between the values for Byrd and GRIP cores $\left(I=4.66 \times 10^{-3} \mathrm{ka}^{-2}\right)$ and the Vostok core $\left(I=3.94 \times 10^{-5} \mathrm{ka}^{-2}\right)$; the illustrations adopt $I^{\star}=$ $10^{-4} \mathrm{ka}^{-2}$. Define a constitutive function, $\chi(I)$, by

$$
\chi(I)=1-\delta\left[1-\tanh \left(\frac{I-I^{\star}}{I^{\star}}\right)\right],
$$

with the properties

$$
\chi(I) \approx 1\left(I \gg I^{\star}\right), \quad \chi(I) \approx 1-2 \delta\left(I \ll I^{\star}\right),
$$

and change the growth relation (Equation (36)) to

$$
\frac{\mathrm{d} \bar{D}}{\mathrm{~d} \tilde{t}}=\frac{\kappa[\bar{T}(\bar{z})]}{2 D_{0}^{2} \bar{D}}[1-\chi(l) g(\bar{D})],
$$

where $I$ is defined by Equation (38), with $s=s_{0} \tilde{S}\left[s_{0}\left(\tilde{t}+t_{\mathrm{p}}\right)\right]$. Now $\chi(l)$ is very close to unity for the Byrd and GRIP cores, 


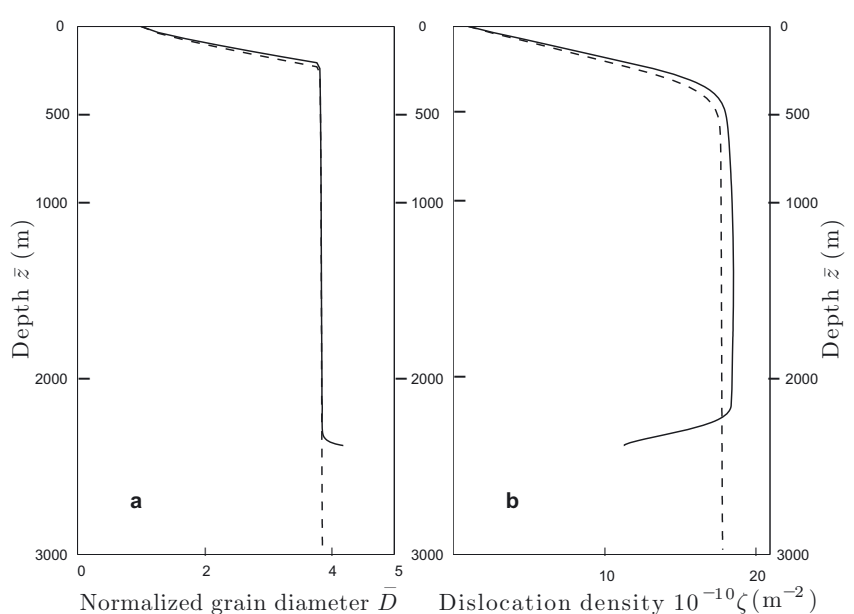

Fig. 10. Same as Figure 9, but for GRIP core.

and to $1-2 \delta$ for the Vostok core, so the grain growths for Byrd and GRIP are not changed by the $\chi(I)$ factor, but for Vostok the growth relation becomes

$$
\text { Vostok : } \quad \frac{\mathrm{d} \bar{D}}{\mathrm{~d} \tilde{t}}=\frac{K[\bar{T}(\bar{z})]}{2 D_{0}^{2} \bar{D}}[1-(1-2 \delta) g(\bar{D})],
$$

the $[1-(1-2 \delta) g(\bar{D})]$ factor is always positive. The parameter $\delta=6 \times 10^{-4}$ was adopted so that $\bar{D} \approx 8$ at the core base, to match figure A2 of De La Chapelle and others (1998).

Expressing the growth relation (41) back in physical variables introduces a possible dependence on the initial value, $D_{0}$, through $g(\bar{D})$, and in the adopted form (Equation (37)), there is dependence on $d\left(D-D_{\mathrm{e}}\right) / D_{0}$, counter to the conclusions of Gao and Jacka (1987) and Jacka and Li (1994). This dependence can be eliminated by setting $d=D_{0} \bar{d}$ with constant $\bar{d}$, when $d$ is not constant, but depends on $D_{0}$, and $g(\bar{D})$ depends explicitly on $D_{\mathrm{e}}$, and not on $\bar{D}_{\mathrm{e}}$. Since $d$ is simply a large parameter introduced in Equation (37) to define a small range of rapid change around $D_{\mathrm{e}}$, its modest change with $D_{0}$ would not significantly change the present results calculated with the large constant $d=100$ in Equation (37).

The dislocation-density evolution given by Equation (35) is retained for both the new and previous correlations. Note that in both evolution equations (35) and (41) the influence of changing flow and profile history arises through the terms $\bar{T}(\bar{z})$ and $\tilde{s}\left[s_{0}\left(\tilde{t}+t_{\mathrm{p}}\right)\right]$, which depend on the time each ice particle has spent in the core.

\section{NUMERICAL ILLUSTRATIONS}

Differential equations (41) and (35) are solved simultaneously for a sequence of surface deposit times, $t_{\mathrm{p}}$, covering the age-depth correlation range, to the present time, $t=0$. The grain-growth equation (41) with adopted constitutive functions (37) and (39) is integrated over the same range to determine the grain-diameter profiles in $\bar{z}$ at $t=0$ for the three cores, both for the new correlation and for the previous correlation with given $s_{0}$ and constant $K$. The function $g(\bar{D})$,

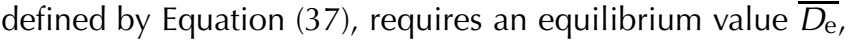
and this was chosen to be 4 , consistent with the values of $D_{\mathrm{e}}$ and $D_{0}$ for the Byrd and GRIP cores in Table 1. Finally, the original dislocation-density evolution equation (35) was integrated over the same range to determine the profiles

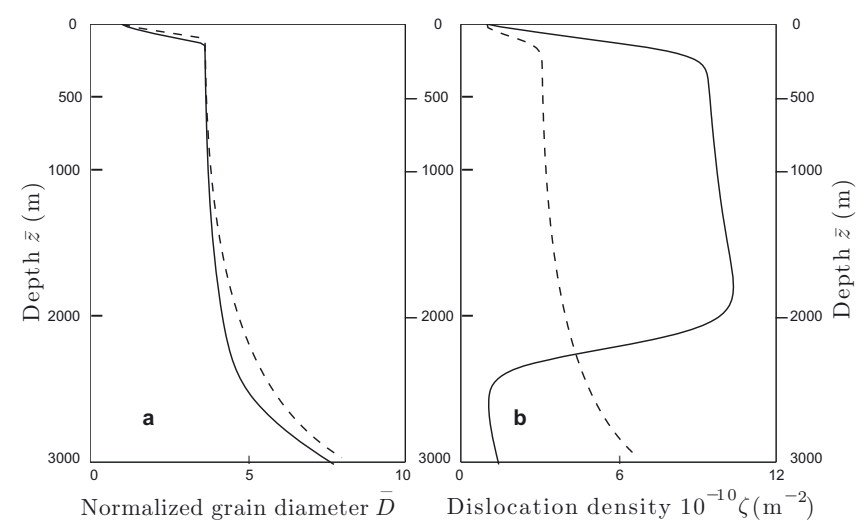

Fig. 11. Vostok core: depth profiles at $t=0$ of (a) normalized grain diameter, $\bar{D}$, and (b) dislocation density, $\zeta$. New correlation solutions (solid curves); solutions for grain growth, Equation (41), with given $s_{0}$ and constant $K$ (dashed curves).

in $\bar{z}$ at $t=0$ for the three cores, both for the new correlation and for the previous correlation with given $s_{0}$ and constant $K$. Accuracy of the numerical algorithm has been checked against equations with exact solutions of similar shape.

The resulting grain-growth profiles for the Byrd and GRIP cores are shown in Figures 9a and 10a. Since the age-depth curves (Figs 2 and 3 ) for the general correlation and constant $s=s_{0}$ are very similar for short age times, the initial normal grain-growth curves are also very similar, but note that the distinct turning point close to the equilibrium diameter arises at a shorter depth than suggested by Montagnat and Duval (2000), even though the initial growth equation, and parameters, are the same. The subsequent range of nearuniform $\bar{D}$ is an observation by Montagnat and Duval (2000), not given by a growth relation. Since temperature remains constant for two-thirds of the core depth, the influence of varying $K(\bar{T})$ in the new correlation only arises near the final depth reached by the correlation in the GRIP core, and not in the Byrd core. Figure 11a shows the resulting grain-growth profiles for the Vostok core for the new and previous correlations. The grain growths for both show a distinct gradient change at $\bar{D}_{\mathrm{e}}$ due to the rapid change of $g(\bar{D})$, which does not occur in figure A2 of De La Chapelle and others (1998). If the latter's smooth variation is correct, the prediction from Equation (34) could be smoothed by reducing parameter $d$ in Equation (37), but if still unsatisfactory, then the growth relation, Equation (34), is not sufficiently general. Even with $K$ constant in the previous correlation solution, but using the growth relation (41), there is still grain growth for $\bar{D}>\bar{D}_{\mathrm{e}}$, where $g(\bar{D})=1$ in Equation (42), since $\delta>0$.

The corresponding dislocation-density profiles in $\bar{z}$ at $t=$ 0 are shown in Figures $9 \mathrm{~b}, 10 \mathrm{~b}$ and $11 \mathrm{~b}$. Montagnat and Duval (2000) assume $D$ remains at $D_{\mathrm{e}}$ for the Byrd and GRIP cores after the initial normal growth, which is the close approximation given by Equation (41), so the dislocationdensity evolution relation, Equation (35), for the two profiles differs only through the strain-rate dependence of the first term. This has made a substantial change in the GRIP core at depths close to the final correlation depth, where the new predicted density decreases rapidly, but also in both cores where the uniform maximum density is much lower in figures 1 and 2 of Montagnat and Duval (2000). The De La Chapelle 
and others (1998) dislocation density in the GRIP core (their fig. 5) has a peak $\sim 18 \times 10^{10} \mathrm{~m}^{-2}$, occurring at $\sim 18 \mathrm{ka}$, corresponding to $\sim 2000 \mathrm{~m}$ depth, and then decreases steadily. Figure $10 \mathrm{~b}$ shows a nearly uniform dislocation-density maximum, $19 \times 10^{10} \mathrm{~m}^{-2}$, from 600 to $2100 \mathrm{~m}$, then a rapid decrease. For the Vostok core, with the grain growth qualitatively similar to figure A2 of De La Chapelle and others (1998), it is clear that a constant $K$ assumption makes a substantial difference to the dislocation-density evolution, but De La Chapelle and others (1998) do not state what variation of $K$ with $T$ has been adopted. Their density has a peak $\sim 13 \times$ $10^{10} \mathrm{~m}^{-2}$ at $50 \mathrm{ka}$, corresponding to a depth $\sim 800 \mathrm{~m}$, then decreases rapidly, very different to the new prediction in Figure $11 \mathrm{~b}$ which shows a peak, $10.8 \times 10^{10} \mathrm{~m}^{-2}$, at $1800 \mathrm{~m}$, then a decrease to $1.1 \times 10^{10} \mathrm{~m}^{-2}$ at $2610 \mathrm{~m}$, followed by an increase to $1.5 \times 10^{10} \mathrm{~m}^{-2}$ at $3000 \mathrm{~m}$.

\section{CONCLUSIONS}

Noting that the assumption of uniform vertical strain rate with depth, together with the assumption of unchanging core conditions over past time, are inconsistent with observed age-depth correlations in the Byrd, GRIP and Vostok cores, the unchanging core history has been relaxed to show how close age-depth correlations can be obtained. Solution of the evolution equations for grain diameter and dislocation density from when each ice particle is deposited at the surface, and interpretation in terms of depth profiles at the present observation time with varying flow and surface/ bed history, requires a consistent depth-time correlation over the period the ice particle has been in the core. The presented correlations may not be appropriate, as suggested by the histories of strain rate obtained, but further information about conditions in the past could be used to change the correlation assumptions and resulting strain-rate and surface/bed histories. However, the correlations provide an illustration of the differences in predictions that can arise. The grain-growth relation has been modified to be consistent with idealized observed profiles, incorporating an illustrative grain-boundary migration rate on temperature, and, in turn, the consequent dislocation-density evolutions are determined. While the new correlation assumptions and grain-growth relations may not be realistic, they demonstrate that a close age-depth correlation leads to results that differ from previous predictions, and that interpretations from core observations hinge on flow-history assumptions.

\section{ACKNOWLEDGEMENTS}

This investigation was prompted by discussions with P. Duval, J. Meyssonnier and R. Starozczyk during a visit to Grenoble in 2002. I wish to thank a referee for very constructive criticisms of my original presentation, and T.H. Jacka for his subsequent guidance, both of which have contributed to an improved presentation.

\section{REFERENCES}

De La Chapelle, S., O. Castelnau, V. Lipenkov and P. Duval. 1998. Dynamic recrystallization and texture development in ice as revealed by the study of deep ice cores in Antarctica and Greenland. J. Geophys. Res., 103(B3), 5091-5105.

Gao, X.Q. and T.H. Jacka. 1987. The approach to similar tertiary creep rates for Antarctic core ice and laboratory prepared ice. J. Phys. [Paris], 48(3), Supplément, 289-295.(Colloq. C1.)

Gow, A.J. 1969. On the rates of growth of grains and crystals in South Polar firn. J. Glaciol., 8(53), 241-252.

Gow, A.J. and T. Williamson. 1976. Rheological implications of the internal structure and crystal fabrics of the West Antarctic ice sheet as revealed by deep core drilling at Byrd Station. Geol. Soc. Am. Bull., 87(12), 1665-1677.

Greve, R. 2001. Glacial isostasy: models for the response of the Earth to varying ice loads. In Straughan, B., R. Greve, H. Ehrentraut and Y. Wang, eds. Continuum mechanics and applications in geophysics and the environment. Berlin, etc., Springer-Verlag, 307-325.

Jacka, T.H. and J. Li. 1994. The steady-state crystal size of deforming ice. Ann. Glaciol., 20, 13-18.

Lipenkov, V.Ya., N.I. Barkov, P. Duval and P. Pimienta. 1989. Crystalline texture of the $2083 \mathrm{~m}$ ice core at Vostok Station, Antarctica. J. Glaciol., 35(121), 392-398.

Montagnat, M. and P. Duval. 2000. Rate controlling processes in the creep of polar ice: influence of grain boundary migration associated with recrystallization. Earth Planet. Sci. Lett., 183(1-2), 179-186.

Morland, L.W. 2002. Influence of lattice distortion on fabric evolution in polar ice. Contin. Mech. Thermodyn., 14(1), 9-24.

Placidi, L., K. Hutter and S.H. Faria. 2006. A critical review of the mechanics of polycrystalline ice. GAMM-Mitt., 29(1), 80-117.

Staroszczyk, R. and L.W. Morland. 2001. Strengthening and weakening of induced anisotropy in polar ice. Proc. $R$. Soc. London, Ser. A, 457(2014), 2419-2440.

Stephenson, P.J. 1967. Some considerations of snow metamorphism in the Antarctic ice sheet in the light of ice crystal studies. In Oura, H., ed. Physics of snow and ice. Sapporo, Hokkaido University. Institute of Low Temperature Science, 725-740.

Thorsteinsson, T., J. Kipfstuhl and H. Miller. 1997. Textures and fabrics in the GRIP ice core. J. Geophys. Res., 102(C12), $26,583-26,599$. 